\title{
EXISTENCE THEOREMS FOR WARFIELD GROUPS
}

\author{
BY
}

\author{
ROGER HUNTER $\left({ }^{1}\right)$, FRED RICHMAN $\left({ }^{2}\right)$ AND ELBERT WALKER $\left({ }^{2}\right)$
}

\begin{abstract}
Warfield studied $p$-local groups that are summands of simply presented groups, introducing invariants that, together with Ulm invariants, determine these groups up to isomorphism. In this paper, necessary and sufficient conditions are given for the existence of a Warfield group with prescribed Ulm and Warfield invariants. It is shown that every Warfield group is the direct sum of a simply presented group and a group of countable torsion-free rank. Necessary and sufficient conditions are given for when a valuated tree can be embedded in a tree with prescribed relative Ulm invariants, and for when a valuated group in a certain class, including the simply presented valuated groups, admits a nice embedding in a countable group with prescribed relative Ulm invariants. These conditions, which are intimately connected with the existence of Warfield groups, are given in terms of new invariants for valuated groups, the derived Ulm invariants, which vanish on groups and fit into a six term exact sequence with the Ulm invariants.
\end{abstract}

1. Introduction. All groups considered in this paper will be abelian, reduced, and $p$-local, that is, multiplication by any prime other than $p$ is an automorphism. In [12] Warfield introduced a class of groups that may be characterized as summands of simply presented groups. Alternatively, they may be thought of as groups that admit a nice decomposition basis with totally projective cokernel. We shall adopt this latter point of view. Warfield showed that a group in this class is determined, up to isomorphism, by its Ulm invariants and invariants that measure how many cyclics of a given type are in a decomposition basis. However he did not give conditions for the existence of groups with prescribed invariants. The utility of having such conditions is illustrated in the case of countable $p$-groups. Ulm's theorem tells you when two groups are isomorphic, but it is only when you also have Zippin's theorem that you can establish various decomposability properties of a group by constructing a direct sum of groups that has the same Ulm invariants. In Theorems 5 and 14 we give the necessary and sufficient conditions for the existence of Warfield groups with prescribed invariants.

Received by the editors July 15, 1976.

AMS (MOS) subject classifications (1970). Primary $20 \mathrm{~K} 99$.

(')This author was partially supported by an Australian National University Postdoctoral Travelling Scholarship.

${ }^{2}$ )These authors were supported by NSF-MPS71-02778-A04.

- American Mathematical Society 1978 
The proof of this theorem also shows that any Warfield group is the direct sum of a simply presented group and a group of countable torsion-free rank.

A valuated group (tree) is a group (tree) equipped with an ordinal valued valuation, where the value of $x$ is usually denoted $v x$. The general existence theorem for Warfield groups rests on two embedding theorems for such valuated structures-the embedding problem is to embed a valuated group (tree) $H$ with a valuation $v$ into a group (tree) $G$ such that $v$ agrees with the valuation induced on $H$ by the $p$-adic valuation of $G$. The first embedding theorem tells when a valuated tree can be embedded in a tree with prescribed Ulm invariants. The second tells when a valuated group (satisfying a mild condition) can be embedded nicely in a countable group with prescribed relative Ulm invariants. This latter theorem generalizes the "fine existence theorem" of Rotman and Yen [8].

All of these theorems are stated in terms of new invariants $g_{A}(\alpha)$. If we consider the Ulm invariant $f_{A}(\alpha)$ to be a functor from valuated groups to vector spaces, then $g_{A}(\alpha+1)$ is its right derived functor. Conversely, $f_{A}(\alpha)$ is the left derived functor of $g_{A}(\alpha+1)$. The functors $g_{A}(\alpha)$ are also defined for limit ordinals $\alpha$. Ulm invariants arise from elements $x$ such that $v x=\alpha$ and $v p x>\alpha+1$. Dually, derived Ulm invariants arise from elements $x$ such that $v x=\alpha$ and, for some $\beta<\alpha$, we cannot write $x=p y$ with $v y>\beta$. Thus the derived Ulm invariants are zero for groups.

2. Valuated trees and groups. A tree is a set $X$, with distinguished element 0 , that admits a multiplication by $p$ satisfying:

(1) $p 0=0$,

(2) $p^{n} x=x$ only if $n=0$ or $x=0$.

An element $x$ of a tree $X$ has order $n$ if $p^{n} x=0$ and $p^{n-1} x \neq 0$. This definition of a tree differs from that given in [2] in that we have not restricted ourselves to torsion trees, that is, trees in which every element has finite order. For a tree $X$ and ordinal $\alpha$, the subset $p^{\alpha} X$ is defined inductively by setting $p^{0} X=X$ and

$$
p^{\alpha} X=\bigcap_{\beta<\alpha} p\left(p^{\beta} X\right)
$$

when $\alpha>0$. The height $h x$ of an element $x$ in $X$ is $\alpha$ if $x \in p^{\alpha} X \backslash p^{\alpha+1} X$. If $x \in p^{\alpha} X$ for all ordinals $\alpha$, then we set $h x=\infty$. The symbol $\infty$ satisfies $\infty<\infty$ and $\alpha<\infty$ for all ordinals $\alpha$.

By a valuated tree we mean a tree $X$ together with a function $v$ on $X$ (called a valuation) such that

(1) $v x$ is an ordinal or $\infty$,

(2) $v p x>v x$.

A valuated tree $X$ is reduced if $v x=\infty$ implies $x=0$. The height function $h$ 
is clearly a valuation satisfying $h x<v x$ for all $x$ in $X$. Any tree is naturally valuated by setting $v x=h x$. Conversely, if $v x=h x$ for all $x$, we say that the valuated tree $X$ is a tree. A map $f: X \rightarrow Y$ of valuated trees is a function such that:

(1) $f(p x)=p f(x)$,

(2) $v f(x) \geqslant v x$.

An embedding of valuated trees is a one-to-one map $f$ such that $v f(x)=v x$ for all $x$. If the inclusion $X \subseteq Y$ is an embedding, we say that $X$ is a valuated subtree of $Y$. If $X$ is a valuated tree, and $\alpha$ is an ordinal, we set

$$
X(\alpha)=\{x \in X: v x>\alpha\} .
$$

A valuated group is a group that is a valuated tree and satisfies

$$
v(x+y) \geqslant \min (v x, v y) \text {. }
$$

It follows that $v n x=v x$ if $p$ does not divide $n$. A map of valuated groups is a group homomorphism that is a map of valuated trees. A valuated group which is a tree is called a group. The valuated groups form a category $\mathfrak{V}_{p}$. An embedding $f: A \rightarrow B$ of valuated groups is a map that is an embedding of valuated trees. If the inclusion map $A \subseteq B$ is an embedding, we say that $A$ is a valuated subgroup of $B$.

The category $\mathfrak{T}_{p}$ is preabelian (additive with kernels and cokernels) and the theory of [5] and [6] provides a natural definition of Ext. A sequence $0 \rightarrow A \rightarrow B \rightarrow C \rightarrow 0$ of valuated groups is in Ext if and only if $0 \rightarrow A(\alpha) \rightarrow$ $B(\alpha) \rightarrow C(\alpha) \rightarrow 0$ is an exact sequence of abelian groups for each $\alpha$. If $A \subseteq B$ is an inclusion of valuated groups, then an element $b$ of $B$ is $A$-proper if $b$ has maximal value among the elements in the coset $b+A$. In case each coset of $A$ contains an element of maximal value, $A$ is said to be nice. It is not difficult to see that $0 \rightarrow A \rightarrow B \rightarrow C \rightarrow 0$ is in Ext if and only if the inclusion $A \subseteq B$ is a nice embedding.

As in [2] we associate with each valuated tree $X$ a valuated group $S(X)$ in the following way. Let $Z_{p}$ denote the integers localized at $p$ and let $F_{X}=\Sigma Z_{p}\langle x\rangle$ be the free $Z_{p}$-module on the nonzero elements of $X$. Let $R_{X}$ be the submodule of $F_{X}$ generated by elements of the form

$$
\begin{array}{ll}
p\langle x\rangle & \text { where } p x=0, \\
p\langle x\rangle-\langle p x\rangle & \text { where } p x \neq 0,
\end{array}
$$

and set $S(X)=F_{X} / R_{X}$. Each element of $S(X)$ can be written in the form $s=\sum u_{i} x_{i}$ where the $u_{i}$ 's are units in $Z_{p}$. Setting $v s=\min v x_{i}$ makes $S(X)$ into a valuated group. This valuated group is called the simply presented valuated group on $X$. If a valuated group $A$ is isomorphic to $S(X)$, then the image of $X$ in $A$ is said to be a spanning tree of $A$.

If $\left\{X_{i}\right\}$ is a family of valuated trees, the direct sum of the $X_{i}$ is their disjoint 
union with zeros identified. If $\left\{A_{i}\right\}$ is a family of valuated groups, the direct sum of the $A_{i}$ is their group direct sum with the value of any element being the minimum value of its components. Note that the valuated group associated with the direct sum of the $X_{i}$ is the direct sum of the valuated groups associated with the $X_{i}$.

3. Derived Ulm invariants. Let $A$ be a valuated group and $\alpha$ an ordinal. Multiplication by $p$ induces a natural map

$$
A(\alpha) / A(\alpha+1) \stackrel{p}{\rightarrow} A(\alpha+1) / A(\alpha+2) .
$$

We denote the kernel and cokernel of this map by $f_{A}(\alpha)$ and $g_{A}(\alpha+1)$, respectively. Both $f_{A}(\alpha)$ and $g_{A}(\alpha+1)$ are vector spaces over the $p$-element field. Clearly

$$
\mathbf{f}_{A}(\alpha)=\frac{\{a \in A(\alpha): p a \in A(\alpha+2)\}}{A(\alpha+1)}
$$

and

$$
\mathbf{g}_{A}(\alpha+1)=\frac{A(\alpha+1)}{(A(\alpha+2)+p A(\alpha))}
$$

where $p A(\alpha)$ denotes $p(A(\alpha))$, a convention that will be adhered to throughout this paper. We shall also need a definition of $g_{A}(\alpha)$ for limit ordinals. Define

$$
\mathbf{g}_{A}(\alpha)=\frac{A(\alpha)}{\bigcap_{\beta<\alpha}(A(\alpha+1)+A(\alpha) \cap p A(\beta))}
$$

This definition agrees with the other for nonlimit ordinals. We call $f_{A}(\alpha)$ the $\alpha$ th Ulm invariant of $A$ and $g_{A}(\alpha)$ the $\alpha$ th derived Ulm invariant of $A$. The dimension of $f_{A}(\alpha)$ will be denoted $f_{A}(\alpha)$. This convention will also be used for all other vector. space invariants occurring in this paper.

If $A$ is a group, $f_{A}(\alpha)$ agrees with the usual definition of Ulm invariant while $g_{A}(\alpha)$ vanishes for all $\alpha$.

We turn now to the relationship between these two invariants. Let $0 \rightarrow A$ $\rightarrow B \rightarrow C \rightarrow 0$ be a sequence in Ext. Then

$$
0 \rightarrow \frac{A(\alpha)}{A(\alpha+1)} \rightarrow \frac{B(\alpha)}{B(\alpha+1)} \rightarrow \frac{C(\alpha)}{C(\alpha+1)} \rightarrow 0
$$

is an exact sequence of vector spaces for each $\alpha$. We arrive at the following commutative diagram: 


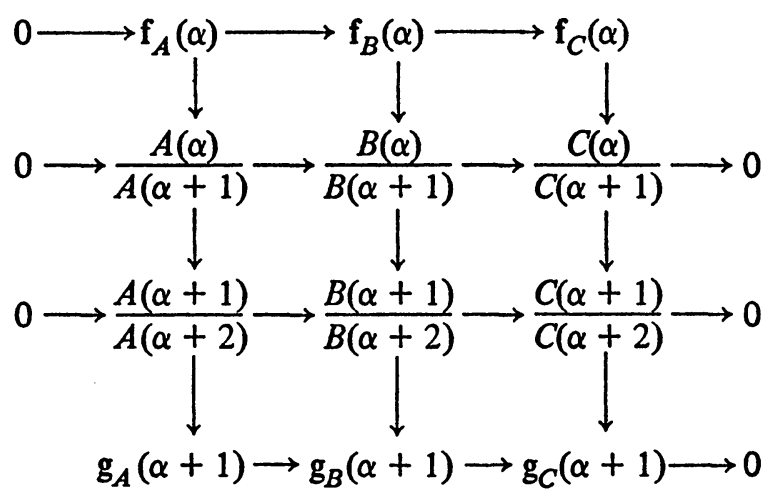

The snake lemma applied to this diagram gives the exact sequence

$$
0 \rightarrow \mathbf{f}_{A}(\alpha) \rightarrow \mathbf{f}_{B}(\alpha) \rightarrow \mathbf{f}_{C}(\alpha) \rightarrow \mathbf{g}_{A}(\alpha+1) \rightarrow \mathbf{g}_{B}(\alpha+1) \rightarrow \mathbf{g}_{C}(\alpha+1) \rightarrow 0 .
$$

This is the fundamental sequence relating the $f$ 's to the $g$ 's. Now $f_{A}$ vanishes when $A$ is projective in $\mathfrak{V}_{p}$, and $\mathbf{g}_{A}$ vanishes on groups. Furthermore, there are enough projectives in $\Upsilon_{p}$, and each $A \in \mathfrak{V}_{p}$ has a nice embedding in a group (for details see [6]). Using this it is easy to show that $f_{A}(\alpha)$ is the left derived functor of $g_{A}(\alpha+1)$, and that $g_{A}(\alpha+1)$ is the right derived functor of $f_{A}(\alpha)$.

For an arbitrary, not necessarily nice, embedding $A \subseteq B$ of valuated groups, the embedding $\phi: f_{A}(\alpha) \rightarrow f_{B}(\alpha)$ is still defined. The cokernel of $\phi$ is denoted $\mathbf{f}_{B, A}(\alpha)$ and called the $\alpha$ th Ulm invariant of $B$ relative to $A$. It is straightforward to check that if $B$ is a group, then this definition agrees with the usual one. It follows that

$$
f_{B}(\alpha)=f_{A}(\alpha)+f_{B, A}(\alpha) .
$$

We derive two further relations of interest. Let $A$ be a nice valuated subgroup of the group $B$. Then $\mathbf{g}_{B}(\alpha+1)=0$ so the exact sequence

$$
0 \rightarrow f_{A}(\alpha) \rightarrow f_{B}(\alpha) \rightarrow f_{B / A}(\alpha) \rightarrow g_{A}(\alpha+1) \rightarrow 0
$$

gives the equation

$$
f_{B / A}(\alpha)=f_{B, A}(\alpha)+g_{A}(\alpha+1) .
$$

Adding $f_{A}(\alpha)$ to each side of this equation, and using the previous equation, gives

$$
f_{B / A}(\alpha)+f_{A}(\alpha)=f_{B}(\alpha)+g_{A}(\alpha+1) .
$$

4. Embedding valuated trees. We define the Ulm and derived Ulm invariants of a valuated tree $X$ to be those of the associated simply presented valuated group $S(X)$. If $X$ is a valuated subtree of $Y$, then $S(X) \subseteq S(Y)$ is a nice embedding and the Ulm invariants of $Y$ relative to $X$ are defined to be the Ulm invariants of $S(Y)$ relative to $S(X)$. The notation introduced for 
these invariants in the preceding section will be carried over unchanged to valuated trees.

We now show how these invariants can be read directly from the valuated trees themselves. Let $Y$ be a valuated tree and $X$ a valuated subtree of $Y$. For each ordinal $\alpha$, set $U_{Y, X}(\alpha)=\{y \in Y \backslash X: v y=\alpha$ and $v p y>\alpha+1\}$. For each $y$ in $p Y(\alpha)$ of value $\alpha+1$, choose an element $z_{y}$ in $Y(\alpha)$ such that $p z_{y}=y$ where $z_{y} \in X$ if possible. Define

$$
D_{Y, X}(\alpha)=\left\{y \in Y(\alpha) \backslash X(\alpha): v p y=\alpha+1 \text { and } y \neq z_{p y}\right\} .
$$

Then we claim that

$$
f_{Y, X}(\alpha)=\left|U_{Y, X}(\alpha)\right|+\left|D_{Y, X}(\alpha)\right|,
$$

and $f_{Y}(\alpha)$ is obtained by choosing $X$ to be trivial. The proof is similar to that of [7, Proposition 2] and involves showing that, for each $\alpha$, the sets $U_{Y, X}(\alpha)$ and $\left\{y-z_{p y}: y \in D_{Y, X}(\alpha)\right\}$ together constitute an irredundant set of representatives of a basis for $f_{Y, X}(\alpha)=f_{S(Y)}(\alpha) / f_{S(X)}(\alpha)$. Similarly, the $\alpha$ th derived Ulm invariant $g_{Y}(\alpha)$ is the number of elements $y$ of $Y$ with value $\alpha$ such that there is $\beta<\alpha$ with $y \notin p Y(\beta)$.

We wish to decide when a valuated tree $X$ can be embedded in a tree $Y$ with specified relative Ulm invariants. In order to do this we need some information concerning functions from ordinals to cardinals which vanish beyond some ordinal. Let $f$ and $g$ be two such functions. We say that $f$ dominates $g$ if

$$
\sum_{n<\omega} f(\alpha+n) \geqslant \sum_{\alpha+\omega<\beta} g(\beta)
$$

for all ordinals $\alpha$. A function which dominates itself is said to be admissible.

THEOREM 1. If $Y$ is a tree, and $X$ is a valuated subtree of $Y$, then $f_{Y, X}$ dominates $f_{Y, X}+g_{X}$.

Proof. Let $Z$ be the subset of $X$ consisting of those elements $z$ for which there is $\beta<v z$ such that $z \notin p X(\beta)$. Then $g_{X}(\alpha)=|\{z \in Z: v z=\alpha\}|$. Define a tree $W$ on the set $(Y \backslash X) \cup Z$ by setting $p y=0$ if $y \in Z$ or $p y \in X \backslash Z$, leaving all other relations unchanged. Thus $W$ is the direct sum of trees $V_{z}$ where $z \in Z$ and $V_{z}$ contains $z$. Letting $z$ also denote the valuated subtree of $V_{z}$ with elements $z$ and 0 , it is readily checked that

$$
f_{Y, X}=f_{W, Z}=\sum_{z \in Z} f_{V_{z}, z^{*}}
$$

Clearly $f_{V_{z}, z}$ dominates $f_{V_{z}, z}+g_{z}$ since $f_{V_{z}}=f_{V_{z}, z}+g_{z}$ is admissible, and $f_{V_{s, z}}=f_{V_{s}}$ at all but one ordinal. Thus

$$
f_{Y, X}=\sum_{z \in Z} f_{V_{z}, z} \text { dominates } \sum_{z \in Z} f_{V_{z}, z}+g_{z}=f_{Y, X}+g_{X} .
$$


The main result of this section is the converse of Theorem 1 . We will need the following lemma.

LEMMA 2. Let $f$ and $g$ be functions from ordinals to cardinals which vanish beyond some ordinal. If $f$ dominates $f+g$, and $g=\Sigma_{l} g_{i}$, then $f$ can be written $f=\Sigma_{I} f_{i}$ such that $f_{i}$ dominates $f_{i}+g_{i}$ for all $i \in I$.

Proof. If $F$ is any function from ordinals to cardinals, and $\alpha$ is an ordinal, define

$$
\phi_{\alpha}(F)=\inf _{k<\omega}\left\{\sum_{n<\omega} F(\alpha+k+n)\right\} .
$$

If $J$ is a nonempty index set, and $\phi_{\alpha}(F) \geqslant|J|$, then it is easy to see that we can write $F=\Sigma_{J} F_{j}$ where $\phi_{\alpha}(F)=\phi_{\alpha}\left(F_{j}\right)$ for all $j$. Let 0 be a distinguished element of $I$ and, for each limit ordinal $\alpha$, define

$$
f^{\alpha}(\beta)= \begin{cases}f(\beta) & \text { if } \alpha \leqslant \beta<\alpha+\omega, \\ 0 & \text { otherwise }\end{cases}
$$

and

$$
\begin{aligned}
I_{\alpha}=\{i \in I: & i \neq 0 \text { and } \phi_{\alpha}(f) \neq 0, \\
& \left.\quad \text { or } g_{i}(\beta) \neq 0 \text { for some } \beta \geqslant \alpha+\omega\right\} .
\end{aligned}
$$

Then $\phi_{\alpha}\left(f^{\alpha}\right)=\phi_{\alpha}(f) \geqslant \Sigma_{\alpha+\omega<\beta} g(\beta)$ so $\phi_{\alpha}\left(f^{\alpha}\right) \geqslant\left|I_{\alpha}\right|$ for all $\alpha$. Therefore, if $\phi_{\alpha}(f) \neq 0$, we can write

$$
f^{\alpha}=\sum_{i \in I_{\alpha}} f_{i}^{\alpha}
$$

where $\phi_{\alpha}\left(f_{i}^{\alpha}\right)=\phi_{\alpha}\left(f^{\alpha}\right)$ for $i \in I_{\alpha}$. Let $\lambda$ be the smallest (limit) ordinal such that $\phi_{\lambda}(f)=0$. Set $f_{0}^{\lambda}=f^{\lambda}$. For all other pairs $\alpha$ and $i$ set $f_{i}^{\alpha}=0$ if $i \notin I_{\alpha}$. Then $f_{i}=\Sigma_{\alpha} f_{i}^{\alpha}$ are the required functions since $\Sigma_{i} f_{i}=\Sigma_{i} \Sigma_{\alpha} f_{i}^{\alpha}=f^{\lambda}+$ $\Sigma_{\alpha} \Sigma_{I_{\alpha}} f_{i}^{\alpha}=\Sigma_{\alpha} f^{\alpha}=f$, and $\phi_{\alpha}\left(f_{i}\right)=\phi_{\alpha}(f)$ if $g_{i}(\beta) \neq 0$ for some $\beta \geqslant \alpha+\omega$, or if $i=0$.

THEOREM 3. Let $X$ be a valuated tree. If $f$ is a function from ordinals to cardinals which vanishes beyond some ordinal and dominates $f+g_{X}$, then $X$ can be embedded in a tree $Y$ such that $f_{Y, X}=f$ and $Y / X$ is torsion.

Proof. Let $Z=\{z \in X: z \notin p X(\beta)$ for some $\beta<v z\}$, and define, for $z \in Z$,

$$
g_{z}(\alpha)= \begin{cases}1 & \text { if } \alpha=v z \\ 0 & \text { otherwise }\end{cases}
$$

By Lemma 2, we may write $f=\Sigma_{z} f_{z}$ so that $f_{z}$ dominates $f_{z}+g_{z}$ for each $z \in Z$. Now $f_{z}+g_{z}$ is admissible for each $z \in Z$, so using a well-known 
property of admissible functions we may write $f_{z}+g_{z}=k_{z}^{1}+k_{z}^{2}$ where $k_{z}^{1}$ and $k_{z}^{2}$ are admissible, $k_{z}^{1}(v z)=1$ and $k_{z}^{1}(\alpha)=0$ for $\alpha>v z$. By a theorem of Crawley and Hales [1, Theorem 83.6] there are torsion trees $V_{z}^{1}$ and $V_{z}^{2}$ with Ulm functions $k_{z}^{1}$ and $k_{z}^{2}$, respectively. Let $V_{z}$ be the direct sum of $V_{z}^{1}$ and $V_{z}^{2}$. Then $V_{z}$ contains an element $z^{\prime}$ with $p z^{\prime}=0$ and $v z^{\prime}=v z$. Notice that $f_{V_{2}, z^{\prime}}=f_{V_{z}}-g_{z}=f_{z}$. Define $Y$ to be the tree obtained from $X$ and the trees $V_{z}$ by identifying $z$ with $z^{\prime}$ for each $z$ in $Z$, and removing the relations $p z^{\prime}=0$. Then $X \subseteq Y$ is an embedding, $Y / X$ is torsion, and $f_{Y, X}=\sum f_{V_{x}, z^{\prime}}=$ $\sum f_{z}=f$.

5. Warfield groups. We begin by extending to valuated groups an invariant introduced by Warfield [11] and generalized by Stanton [9]. Our approach parallels that of Stanton. The value sequence of an element $x$ is the sequence $\left(v x, v p x, v p^{2} x, \ldots\right)$. Any sequence $\mu=\left(\mu_{0}, \mu_{1}, \ldots\right)$ of ordinals and symbols $\infty$ such that $\mu_{i}<\mu_{i+1}$ for $i=0,1, \ldots$ will be called a value sequence, and the sequence $\left(\mu_{1}, \mu_{2}, \ldots\right)$ will be denoted by $p \mu$. Value sequences $\mu$ and $\nu$ are equivalent if there exist $m$ and $n$ such that $p^{m} \mu=p^{n} v$. Let

$$
A(\mu)=\left\{x \in A: v p^{k} x \geqslant \mu_{k} \text { for all } k\right\}
$$

and let $A(\mu)^{*}$ be the valuated subgroup of $A$ generated by those elements $x$ of $A(\mu)$ such that $v p^{k} x \neq \mu_{k}$ for infinitely many $k$. Multiplication by $p$ induces natural maps

$$
A(\mu) / A(\mu)^{*} \rightarrow A(p \mu) / A(p \mu)^{*} \rightarrow A\left(p^{2} \mu\right) / A\left(p^{2} \mu\right)^{*} \rightarrow \cdots
$$

which are one-to-one [9, Lemma 1]. When $A$ is a group, these maps are all isomorphisms. The direct limit of this system is a vector space over the $p$-element field and depends only on the equivalence class of $\mu$. We call this direct limit the Warfield invariant of $A$ at $\mu$ and denote it $\mathbf{w}_{A}(\mu)$.

LEMMA 4. Let $A \subseteq B$ be an embedding of valuated groups. If $B / A$ is torsion, then $\mathbf{w}_{A}(\mu)=\mathbf{w}_{B}(\mu)$ for all value sequences $\mu$.

Proof. The embedding $A \subseteq B$ induces a natural map $\phi: w_{A}(\mu) \rightarrow \mathbf{w}_{B}(\mu)$. On the other hand, if $x \in B\left(p^{n} \mu\right)$, then $p^{m} x \in A$ for some $m$, so $p^{m} x \in$ $A\left(p^{m+n} \mu\right)$ represents an element of $\mathrm{w}_{A}(\mu)$. This allows us to define a map $\psi: w_{B}(\mu) \rightarrow w_{A}(\mu)$ in the obvious way. It is readily checked that $\phi$ and $\psi$ are inverses of each other.

Let $A$ be a valuated group and $H$ a valuated subgroup of $A$. If $H$ is a (valuated) direct sum of cyclics, and $A / H$ is torsion, then a basis $X$ for $H$ is called a decomposition basis for $A$. We sometimes refer to $H$ itself as a decomposition basis for $A$; the context will always make clear which is meant. For each value sequence $\mu$ we have 


$$
\begin{aligned}
w_{A}(\mu) & =w_{H}(\mu) \\
& =\mid\left\{x \in X:\left(v x, v p x, v p^{2} x, \ldots\right) \text { is equivalent to } \mu\right\} \mid .
\end{aligned}
$$

If $H$ is nice in $A$ and $A / H$ is simply presented, then $A$ is called a Warfield group. Warfield [12] has shown that these groups are precisely the summands of simply presented groups and has proved that two Warfield groups are isomorphic if and only if they have the same Ulm invariants and the same Warfield invariants.

The only thing that keeps a Warfield group from being simply presented is a lack of relative Ulm invariants. The following theorem makes this precise.

Theorem 5. Let $G$ be a Warfield group and $X$ a decomposition basis of $G$ generating a nice subgroup $H$ with simply presented cokernel. Then $G$ is simply presented, with spanning tree containing $X$ if and only if $f_{G, H}$ dominates $f_{G, H}+g_{H}$.

Proof. Suppose $X$ can be embedded in a tree $Y$ such that $G=S(Y)$. Then $f_{Y, X}$ dominates $f_{Y, X}+g_{X}$ by Theorem 1. Since $f_{G, H}=f_{Y, X}$ and $g_{H}=g_{X}$ we have proved the "only if". Conversely, if $f_{G, H}$ dominates $f_{G, H}+g_{X}$, then, by Theorem 3, there exists an embedding of $X$ in a tree $Y$ with $f_{G, H}=f_{Y, X}$. Since $f_{G}=f_{G, H}+f_{H}=f_{Y, X}+f_{X}=f_{Y}$, we can apply Hill's theorem [10, Theorem 2.8] to get an isomorphism of $G$ with $S(Y)$ that is the identity on $X$, taking care of the "if".

An easy consequence of Theorem 5 is the well-known fact that any Warfield group is a summand of a simply presented group with a simply presented torsion complement.

Let $X$ and $Y$ be decomposition bases. Following Warfield we say $Y$ is subordinate to $X$ if each element of $Y$ is a multiple of an element in $X$. If $X$ generates a nice subgroup so does $Y$, and, using the characterization of simply presented $p$-groups as those having a nice composition series [1, Theorems 82.3 and 83.5], it is easy to see that the cokernel of $Y$ is simply presented if the cokernel of $X$ is.

THEOREM 6. Let $H$ be a nice decomposition basis of a group $G$ with simply presented cokernel. Then $f_{G} \geqslant f_{H}$ and $f_{G}$ dominates $f_{G}+g_{H}$.

Proof. The first assertion follows immediately from the equation $f_{G}=f_{G, H}$ $+f_{H}$. For the second, we first effect a number of reductions. If $K$ is subordinate to $H$, then

$$
\sum_{\lambda<\beta} g_{H}(\beta)=\sum_{\lambda<\beta} g_{K}(\beta)
$$

if either side is infinite or 0 , for all limit ordinals $\lambda$. Thus it suffices to prove the theorem for some basis subordinate to $H$. For each ordinal $\alpha$, the group 
$p^{\alpha} G$ is Warfield. Thus, in order to verify that

$$
\sum_{n<\omega} f_{G}(\alpha+n) \geqslant \sum_{\alpha+\omega<\beta} f_{G}(\beta)+g_{H}(\beta)
$$

for each $\alpha$, and each Warfield group $G$, we can and will assume $\alpha<\omega$.

By passing to a subordinate basis if necessary, we arrange that each basis element $x$ of $H$ has no gaps or infinitely many gaps in its value sequence, that $v x<\omega$ implies $v p^{n} x<\omega$ for all $n$, and that $v x \neq \omega$. In particular,

$$
\sum_{\omega<\beta} g_{H}(\beta+1)=\sum_{\omega<\beta} g_{H}(\beta)
$$

and $\Sigma_{\theta<\beta} f_{H}(\beta)=0$ or is infinite for all $\theta$. Let $K$ be generated by those basis elements of $H$ with value less than $\omega$ whose value sequences have no gaps. Let $\phi$ be the natural map $G \rightarrow G / p^{\omega} G$. Then $\phi K$ is a nice decomposition basis for $G / p^{\omega} G$ with simply presented cokernel, so Theorem 5 shows that $G / p^{\omega} G$ is simply presented with spanning tree containing the given basis of $\phi H$. We may write $G / p^{\omega} G=L \oplus M$ where $\phi K$ is a nice decomposition basis for $L$ with simply presented cokernel. By Hill's theorem [10, Theorem 2.8] we can write $L=F \oplus C$ where $C$ is a direct sum of finite cyclics, $F$ is a free group, and $\phi K$ is subordinate to $F$. Then $G$ contains a free group $F^{\prime}$ that maps isomorphically onto $F$ under $\phi$, and $G=F^{\prime} \oplus \phi^{-1}(C \oplus M)$. It is easy to check that $\phi^{-1}(C \oplus M)$ is Warfield. Since $F^{\prime}$ does not contribute to the domination calculations, we may assume $F^{\prime}=0$. With this final reduction, each basis element $x$ in $H$ with $v x<\omega$ has infinitely many gaps in its value sequence and therefore

$$
\sum_{n<\omega} \cdot f_{H}(k+n)=\sum_{n<\omega} g_{H}(k+n+1)
$$

for all $k<\omega$. But then

$$
\begin{aligned}
\sum_{n<\omega} f_{G / H}(k+n) & =\sum_{n<\omega} f_{G, H}(k+n)+g_{H}(k+n+1) \quad \text { by }(*) \\
& =\sum_{n<\omega} f_{G, H}(k+n)+f_{H}(k+n)=\sum_{n<\omega} f_{G}(k+n)
\end{aligned}
$$

for all $k<\omega$. Now $G / H$ is simply presented, so $f_{G / H}$ is admissible. Hence

$$
\begin{aligned}
\sum_{n<\omega} f_{G}(k+n) & =\sum_{n<\omega} f_{G / H}(k+n) \geqslant \sum_{\omega<\beta} f_{G / H}(\beta) \\
& =\sum_{\omega<\beta} f_{G, H}(\beta)+g_{H}(\beta+1) \text { by }(*) \\
& =\sum_{\omega<\beta} f_{G, H}(\beta)+g_{H}(\beta) \\
& =\sum_{\omega<\beta} f_{G, H}(\beta)+f_{H}(\beta)+g_{H}(\beta)
\end{aligned}
$$


as $\sum_{\theta<\beta} g_{H}(\beta) \geqslant \sum_{\theta \leqslant \beta} f_{H}(\beta)$ for all $\theta$. The latter equation expresses the fact that, for a direct sum of cyclics, $f$ counts the bottoms of the gaps and $g$ counts the tops. Now $\Sigma_{\omega<\beta} f_{G, H}(\beta)+f_{H}(\beta)+g_{H}(\beta)=\Sigma_{\omega<\beta} f_{G}(\beta)+g_{H}(\beta)$. This completes the proof.

Note that if $H$ is a direct sum of cyclics, then $\Sigma_{\alpha+\omega<\beta} g_{H}(\beta)$ is determined, to within a finite cardinal, by the Warfield invariants of $H$. In the preceding theorem, the Warfield invariants of $G$ and $H$ are the same so the fact that $f_{G}$ dominates $f_{G}+g_{H}$ can therefore be interpreted as a statement connecting the Ulm and Warfield invariants of $G$.

COROLlaRY 7. If $G$ is a Warfield group, then the direct sum of infinitely many copies of $G$ is simply presented.

Proof. It suffices to look at the countable case. Let $A=\Sigma G_{n}$ where each $G_{n}$ is isomorphic to $G$. Let $X$ be a nice decomposition basis of $G$ with simply presented cokernel, and let $X_{n}$ be the corresponding decomposition basis of $G_{n}$. Then $Y=U p^{n} X_{n}$ is a nice decomposition basis of $A$ with simply presented cokernel, and $f_{A, Y}=f_{A}$. But $f_{A}$ dominates $f_{A}+g_{Y}$ by Theorem 6 . Hence $f_{A, Y}$ dominates $f_{A, Y}+g_{Y}$, so $A$ is simply presented by Theorem 5 .

We may use this corollary to provide a simple example of a nice decomposition basis $X$ of a simply presented group, with a simply presented cokernel, such that $X$ cannot be extended to a spanning tree. Let $G$ be a Warfield group with decomposition basis $x$ such that the value sequence of $x$ is $(0,2,4, \ldots)$ and $f_{G, x}(\alpha)=1$ if $\alpha=\omega$, and 0 otherwise. Such a group exists by the "fine existence theorem" of Rotman and Yen [8]. Warfield [12, Example 2.7] pointed out that $G$ is not simply presented, which observation was the inspiration for this paper. However the direct sum $A$ of countably many copies of $G$ is simply presented, by Theorem 6 , whereas the decomposition basis $X$ of $A$, composed of the $x$ 's, cannot be extended to a spanning tree since $f_{A, X}$ is not admissible (Theorem 1 ).

6. The countable case. In this section we show how to construct countable Warfield groups with prescribed invariants (Theorem 13). To do this we address the problem of when a valuated group $H$ can be embedded nicely in a countable group $G$ with prescribed relative Ulm invariants. If $p H(\beta)$ is nice in $H$ for all $\beta$, we get necessary and sufficient conditions (Theorems 11 and 12). This generalizes the "fine existence theorem" of Rotman and Yen [8] which deals with the case when $H$ is cyclic. The condition on $p H(\beta)$ is easily seen to hold for direct sums of cyclics or even simply presented valuated groups. An example shows that this condition cannot be removed.

The fundamental construction step is the following.

LEMMA 8. Let $H$ be a valuated group and $x \in H$. If $\beta<v x$, then $H$ can be 
embedded nicely in a valuated group $K=(H, x, \beta)$ that is generated by $H$ and a distinguished element $y_{K}$, such that $y_{K}$ is $H$-proper, $v y_{K}=\beta$, and $p y_{K}=x$. Any two'such valuated groups are isomorphic over $H$.

Proof. Define $K$ by adjoining an element $y_{K}$ to $H$ subject only to the relation $p y_{K}=x$, and valuate $K$ by setting

$$
v\left(u y_{K}+s\right)=\min (\beta, v s)
$$

if $s \in H$ and $u$ is a unit, and leaving the valuation on $H$ the same. It is readily checked that this does define a valuation on $K$, and that $y_{K} \in K$ has the desired properties. If $L$ is another candidate, the map that is the identity on $H$ and takes $y_{K}$ to $y_{L}$ is easily seen to be an isomorphism.

The derived Ulm invariant $\mathbf{g}_{H}(\mu)$ admits a natural filtration by the subspaces

$$
\mathbf{J}_{H}(\lambda, \mu)=\frac{H(\mu+1)+H(\mu) \cap p H(\lambda)}{\bigcap_{\sigma<\mu}(H(\mu+1)+H(\mu) \cap p H(\sigma))}
$$

for $\lambda<\mu$. Note that if $H$ is a cyclic valuated group, then $\mathbf{J}_{H}(\lambda, \mu) \neq 0$ exactly when there is an $x \in H(\lambda)$ such that $v p x=\mu>v x+1$. The invariants $J_{H}(\lambda, \mu)$ play a crucial role in determining whether $H$ can be embedded in a group $G$ with prescribed relative Ulm invariants. We will need the following facts about the invariants of the valuated group constructed in Lemma 8.

LEMMA 9. Let $H$ be a valuated group and $x$ an element of $H$. If $\beta<v x=\alpha$, then $K=(H, x, \beta)$ satisfies:

(1) $f_{K, H}(\theta)=0$ if $\theta \neq \beta$,

(2) $f_{K, H}(\beta)=0$ if $\alpha=\beta+1$ and $x$ is $p H(\beta)$-proper,

(3) $f_{K, H}(\beta)=1$ if $\alpha>\beta+1$,

(4) $g_{K}(\beta) \neq 0$ if $\beta \neq 0$,

(5) $g_{H}(\theta)$ maps onto $g_{K}(\theta)$ if $\theta>\beta$,

(6) $J_{H}(\lambda, \mu)=J_{K}(\lambda, \mu)$ if $\beta \geqslant \mu$.

Proof. The first statement follows from the exact sequence

$$
0 \rightarrow \mathbf{f}_{H}(\theta)+\mathbf{f}_{K}(\theta) \rightarrow \mathbf{f}_{K / H}(\theta)=0 .
$$

If $y^{\prime} \in K$ represents a nonzero element of $\mathbf{f}_{K, H}(\beta)$, then $y^{\prime}=u y_{K}+z$ for some $z \in H(\beta)$ and some unit $u$. So $\beta+1<v p y^{\prime}=v(u x+p z) \leqslant \alpha$ if $x$ is $p H(\beta)$-proper. On the other hand, if $\alpha>\beta+1$, then $u y_{K}$ represents a nonzero element of $\mathbf{f}_{K, H}(\beta)$, and $\mathbf{f}_{K, H}(\beta)$ is then clearly one-dimensional. For (4) we note that $y_{K} \in K$ represents a nonzero element of $\mathbf{g}_{K}(\beta)$ because $K(\beta+1)+p K \subseteq H$. Statement (5) follows from the fact that $H(\beta+1)=$ $K(\beta+1)$. To prove (6) we use the fact that if $\beta \geqslant \lambda$, then $K(\lambda)=H(\lambda)+$ 
$K(\beta)$, so

$$
\begin{aligned}
K(\mu+1)+K(\mu) \cap p K(\lambda) & =K(\mu+1)+K(\mu) \cap(p H(\lambda)+p K(\beta)) \\
& =K(\mu+1)+H(\mu) \cap p H(\lambda) .
\end{aligned}
$$

Now (6) follows from the observation that each representative of $\mathbf{J}_{K}(\lambda, \mu)$ (respectively $\mathbf{J}_{H}(\lambda, \mu)$ ) can be assumed to lie in $K(\mu) \cap p K \cdot(\lambda)$ (respectively $H(\mu) \cap p H(\lambda))$.

Definition. Let $f$ be a function from ordinals to cardinals and $H$ a valuated group. We say that $H$ admits $f$ at $\theta$ if there are infinitely many ordinals $\lambda$ between $\theta$ and $\theta+\omega$ such that

(1) $f(\lambda) \neq 0$ or

(2) $J_{H}(\lambda, \lambda+n) \neq 0$ for some positive integer $n$.

Lemma 10. Let $H$ be a valuated group, $x$ an element of $H, \beta<v x$, and $K=(H, x, \beta)$. Let $f$ and $f^{\prime}$ be functions on the ordinals such that $f=f^{\prime}$ except possibly at finitely many ordinals. Then the following are equivalent for all ordinals $\theta$ :

(1) $H$ admits $f$ at $\theta$,

(2) $K$ admits $f$ at $\theta$,

(3) $K$ admits $f^{\prime}$ at $\theta$.

Proof. If $\theta+\omega>\beta$, then we may assume that $\theta>\beta$. But $H(\beta+1)=$ $K(\beta+1)$, so $J_{H}(\lambda, \lambda+n)=J_{K}(\lambda, \lambda+n)$ for all $\lambda \geqslant \theta$ and positive integers $n$. If $\theta+\omega \leqslant \beta$, the same is true by Lemma 9, part (6). Thus (1) is equivalent to (2). That (2) is equivalent to (3) is clear.

Definition. Let $H$ be a valuated group and $f$ a function on the ordinals. We say that $H$ admits $f$ if $H$ admits $f$ at each ordinal $\theta$ for which there is $\alpha \geqslant \theta+\omega$ such that $g_{H}(\alpha) \neq 0$ or $f(\alpha) \neq 0$.

THEOREM 11. Let $H$ be a nice valuated subgroup of a reduced group $G$. Then $H$ admits $f_{G, H}$.

Proof. Call two nice valuated subgroups $K$ and $L$ of $G$ equivalent if for all $\theta$,

(1) $H$ admits $f_{G, H}$ at $\theta$ if and only if $K$ admits $f_{G, K}$ at $\theta$, and

(2) $f_{G, H}=f_{G, K}$ except possibly at finitely many ordinals.

If $K$ is nice and $K^{\prime}$ is isomorphic to $(K, x, \beta)$ over $K$, then $K^{\prime}$ is nice and is equivalent to $K$. Let $S$ be the set of ordinals $\sigma$ such that $g_{K}(\sigma) \neq 0$ for some $K$ equivalent to $H$. We shall show that $\beta \in S$ if $f_{G, H}(\beta) \neq 0$ or if $0<\beta<\alpha \in$ $S$.

If $f_{G, H}(\beta) \neq 0$, choose $y \in G$ representing a nonzero element of $\mathbf{f}_{G, H}(\beta)$ such that $p y=0$. Let $K$ be generated by $H$ and $y$. Then $K \cong(H, 0, \beta)$ is equivalent to $H$ and $g_{K}(\beta) \neq 0$, so $\beta \in S$. Suppose $0<\beta<\alpha \in S$. We 
shall show that $\beta \in S$ by induction on $\alpha$. Since $\alpha \in S$ we can find $K$ equivalent to $H$ and $x \in K$ representing a nonzero element of $g_{K}(\alpha)$. If $\alpha=\alpha^{\prime}+1$, choose $y \in G\left(\alpha^{\prime}\right)$ such that $p y=x$ and let $K^{\prime}$ be generated by $K$ and $y$. Then $K^{\prime} \cong\left(K, x, \alpha^{\prime}\right)$ is equivalent to $H$ and $g_{K^{\prime}}\left(\alpha^{\prime}\right) \neq 0$. Thus $\alpha^{\prime} \in S$ and so, by induction, $\beta \in S$. If $\alpha$ is a limit, choose $\beta \leqslant \theta<\alpha$ so that $x$ is $p K(\theta)$-proper. Choose $y \in G(\theta)$ so that $p y=x$ and choose $z \in K$ maximizing $v(y+z)=\beta^{\prime}$. Then $K^{\prime} \cong\left(K, x+p z, \beta^{\prime}\right)$ is equivalent to $H$ and $g_{K^{\prime}}\left(\beta^{\prime}\right) \neq 0$. But $\beta<\beta^{\prime}<\alpha$, since $x$ is $p K(\theta)$-proper and $z \in K(\theta)$, so $\beta \in S$ by induction.

If $g_{H}(\alpha) \neq 0$ or $f_{G, H}(\alpha) \neq 0$ for some $\alpha \geqslant \theta+\omega$, then $\theta+\omega \in S$ so we may let $\alpha=\theta+\omega$ and we are in the situation of the preceding paragraph where $\alpha$ is a limit. If $p z$ represents a nonzero element of $g_{K}\left(\beta^{\prime}+1\right)$, then $p z$ represents a nonzero element of $\mathrm{J}_{K}\left(v z, \beta^{\prime}+1\right)$. If $p z$ does not represent a nonzero element of $\mathbf{g}_{K}\left(\beta^{\prime}+1\right)$, then $v(p z-p u)>\beta^{\prime}+1$ for some $u \in$ $H\left(\beta^{\prime}\right)$, so $y+z-u$ represents a nonzero element of $\mathbf{f}_{G, K}\left(\beta^{\prime}\right)$, whence $f_{G, K}\left(\beta^{\prime}\right) \neq 0$. Since $\theta$ may be replaced by $\theta+m$, this establishes that $K$ admits $f_{G, K}$ at $\theta$, and so $H$ admits $f_{G, H}$ at $\theta$.

The construction of a nice embedding of $H$ in a group $G$ with prescribed relative Ulm invariants proceeds along the lines of the constructive existence theorems for countable $p$-groups and $K T$-modules developed in [3] and [4]. The basic idea is to grow a group on $H$ by sequentially enlarging it, adjoining at each step a solution $y$ to the equation $p y=x$ with $v y>\beta$ for some prescribed $\beta<v x$.

THEOREM 12. Let $H$ be a countable valuated group such that $p H(\theta)$ is nice for all $\theta$, and $v x$ is a countable ordinal for every nonzero element $x$ in $H$. Let $f$ be a function from the ordinals to the countable cardinals which vanishes beyond some countable ordinal. If $H$ admits $f$, then $H$ can be embedded as a nice valuated subgroup of a countable reduced group $G$ such that $G / H$ is torsion and $f_{G, H}=f$.

Proof. We shall construct $G$ as the union (direct limit) of a chain of valuated groups $H=H_{0} \subseteq H_{1} \subseteq H_{2} \subseteq \cdots$ where $H_{n+1}=\left(H_{n}, x_{n}, \alpha_{n}\right)$, the ordinal $\alpha_{n}$ is countable, and $x_{n}$ is $p H_{n}\left(\alpha_{n}\right)$-proper. Before indicating how to choose the $x_{n}$ and $\alpha_{n}$, we shall observe some facts about this situation. Repeated application of Lemma 9 shows that $f_{H_{n}, H}=0$ except for finitely many ordinals. Next we show, by induction on $n$, that $p H_{n}(\theta)$ is nice for every $\theta$. Since $H_{n+1} / H_{n}$ is finite, so are $H_{n+1}(\theta) / H_{n}(\theta)$ and $p H_{n+1}(\theta) / p H_{n}(\theta)$. Since $p H_{n}(\theta)$ is nice in $H_{n}$, and $p H_{n+1}(\theta) \subseteq H_{n}$, we have $p H_{n+1}(\theta)$ is nice in $H_{n}$ since $p H_{n+1}(\theta) / p H_{n}(\theta)$ is finite. But $H_{n}$ is nice in $H_{n+1}$, so $p H_{n+1}(\theta)$ is nice in $H_{n+1}$ as desired. Finally we want to show, by induction on $n$, that $H_{n}$ admits $f$. For this to be true we must put some further 
restrictions on $\alpha_{n}$. We shall assume that either $f\left(\alpha_{n}\right) \neq 0$ or $g_{H_{n}}(\theta) \neq 0$ for some $\theta>\alpha_{n}$. By part (5) of Lemma 9 , if $g_{H_{n+1}}(\lambda) \neq 0$ for some $\lambda>\alpha_{n}$, then $g_{H_{n}}(\lambda) \neq 0$. Thus Lemma 10 implies that $H_{n+1}$ admits $f$ if $H_{n}$ does.

We proceed with the construction. Note that $f_{G, H}=\lim _{n \rightarrow \infty} f_{H_{n}, H}$. We want $f_{H_{n}, H}$ to converge to $f$, so we must keep $f_{H_{n}, H} \leqslant f$. We also want $G$ to be a group, so there are two basic constructions of $H_{n+1}$. The first will assure that $G$ is a group and that $f_{G, H} \leqslant f$. The second will make $f_{G, H}=f$.

Suppose $x \in H_{n}$ and $\beta<v x=\alpha$. We want to be able to write $x=p y$ for some $y \in H_{n+1}(\beta)$. We may assume that $x$ has maximum value in $x+$ $p H_{n}(\beta)$, and that $x \neq 0$. Then $x$ represents a nonzero element of $g_{H_{n}}(\alpha)$. If $\alpha$ is not a limit, let $\alpha_{n}=\alpha-1$ and $x_{n}=x$. Since $f_{H_{n+1}, H_{n}}=0$, by Lemma 9 , we have $f_{H_{n+1}}=f_{H_{n}} \leqslant f$.

Now suppose $\alpha$ is a limit. Notice that $H_{n}$ admits $f-f_{H_{n}, H}$ since $H_{n}$ admits $f$ and $f_{H_{n}, H}=0$ for all but a finite number of ordinals. Since $g_{H_{n}}(\alpha) \neq 0$, there is $\beta<\beta^{\prime}<\beta+\omega$ such that either $f\left(\beta^{\prime}\right)>f_{H_{n}, H}\left(\beta^{\prime}\right)$, in which case we set $\alpha_{n}=\beta^{\prime}$ and $x_{n}=x$, or there is a $z \in H_{n}\left(\beta^{\prime}\right) \backslash H_{n}\left(\beta^{\prime}+1\right)$ and $m>1$ such that $p z \in H_{n}\left(\beta^{\prime}+m\right)$ and $p z \notin H_{n}\left(\beta^{\prime}+m+1\right)+H_{n}\left(\beta^{\prime}+m\right) \cap p H_{n}\left(\beta^{\prime}\right.$ $+m-1)$, in which case we set $x_{n}=x+p z$ and $\alpha_{n}=\beta^{\prime}+m$. In the first case we have $f_{H_{n+1}} \leqslant f$ by Lemma 9 . In the second case, note that $x+p z$ is $p H_{n}\left(\beta^{\prime}+m-1\right)$-proper since $p z$ is $p H_{n}\left(\beta^{\prime}+m-1\right)$-proper and $v p z=\beta^{\prime}$ $+m<\alpha=v x$. Hence $f_{H_{n+1}, H}=0$, by Lemma 9 , so $f_{H_{n+1}}<f$.

The second construction is simply to set $x_{n}=0$ and $\alpha_{n}=\theta$ if $f(\theta)>$ $f_{H_{n}, H}(\theta)$. This makes $f_{H_{n+1}}(\theta)=f_{H_{n}}(\theta)+1$. By alternating these constructions, running through all the $\theta$ an infinite number of times, and all pairs $(\beta, x)$ with $\beta<v x$, including those $x$ that are constructed at earlier stages, we achieve the desired result.

To see the necessity for the condition that $p H(\theta)$ be nice for all $\theta$, let $\xi$ be an irrational $p$-adic integer and let $H$ be the valuated subgroup generated by 1 and $p \xi$. Now $p H$ is not nice in $H$ because we can find $n$ such that $v(n+\xi)$ is arbitrarily large, so $p \xi+p H$ contains no element of maximum value. Let $G$ be a reduced group containing $H$ as a valuated subgroup such that $f_{G, H}=0$. Then $G$ is torsion-free and there is an $x \in G$ such that $p x=p \xi$. We shall show that $x+H$ contains no element of maximum value. Indeed $v(n+x)$ $+1=v(p n+p \xi)=v(n+\xi)+1$ can be made as close to $\omega$ as we wish, while $v(x+z)+1=v(p \xi+p z)<\omega$ for $z \in H$.

As an application of Theorem 12 we show the existence of a Warfield group of infinite torsion-free rank, any summand of which is either finite or of finite index.

EXAMPLE. Let $H=\sum_{n=0}^{\infty} C_{n}$ where $C_{n}$ is generated by $x_{n}$ such that

$$
v p^{m} x_{n}=\omega n+2 m .
$$


Then $H$ can be embedded nicely in a Warfield group $G$ with the same Ulm invariants as $H$, such that $G / H$ is torsion. Any summand of $G$ is either finite or of finite index.

Proof. We have $J_{H}(\omega n+2 m$, $\omega n+2 m+2) \neq 0$, so $H$ admits the zero function, whence the desired $G$ exists by Theorem 12. Suppose $G=A \oplus B$ and let $\mu=(0,2,4, \ldots)$. Since $w_{G}(\mu) \neq 0$ we may assume that $w_{A}(\mu) \neq 0$. Then $A$ must contain an element $y$ such that $v p^{n} y=2 m+2 n$ for some $m$ and all $n$. Hence $f_{A}(2 m+2 n) \neq 0$ for all $n$, so $f_{B}(k)=0$ for all $k \geqslant 2 m$. This implies that the torsion subgroup of $B$ is bounded, and so $B$ is bounded since $G$ admits no nonzero torsion-free summands $\left(w_{G}(0)=0\right)$.

This example shows that a Warfield group of infinite torsion-free rank need not have a summand of nonzero finite torsion-free rank. However, we shall see later that every Warfield group is the sum of a simply presented group and a Warfield group of countable torsion-free rank (Theorem 14). But first we need the fundamental existence theorem for countable Warfield groups.

THEOREM 13. Let $H$ be a countable direct sum of infinite cyclic valuated groups such that $v x$ is a countable ordinal for each nonzero $x \in H$. Let $F$ be a function from the ordinals to the countable cardinals that vanishes beyond some countable ordinal. If $F$ dominates $F+g_{H}$ and $F \geqslant f_{H}$, then there is a valuated subgroup $K$ subordinate to $H$ and admitting $f$ such that $F=f_{K}+f$. Hence there exists a countable Warfield group $G$ with Ulm function $F$ and Warfield invariants equal to those of $H$.

Proof. Write $H=\Sigma_{i<\omega} C_{i}$ where $C_{i}$ is infinite cyclic. Let

$$
B=\left\{\beta \text { : for all } m, f_{C_{i}}(\beta+n) \neq 0 \text { for some } n \geqslant m \text { and } i \geqslant m\right\} \text {. }
$$

We shall construct $K$ subordinate to $H$ that admits $f_{H, K}$ at all $\beta \in B$. Let $\beta_{1}, \beta_{2}, \ldots$ be an enumeration of $B$ in which each element appears an infinite number of items. Inductively choose $i(m)>i(m-1)$ such that $f_{C_{i(m)}}\left(\beta_{m}+\right.$ $n(m)) \neq 0$ where $n(m)>m$. Then $K$ consists of those elements of $H$ whose projection on $C_{i(m)}$ is in $p^{n(m)+1} C_{i(m)}$ for all $m$. Let $f=f_{H, K}+F-f_{H}$ where $f(\alpha)$ is infinite if $F(\alpha)$ is infinite. Note that $F=f_{K}+f$.

We now show that $K$ admits $f$. Suppose $g_{K}(\alpha) \neq 0$ or $f(\alpha) \neq 0$ for some $\alpha>\beta+\omega$. If $\beta \in B$, then $K$ admits $f_{H, K}$ at $\beta$, so $K$ admits $f>f_{H, K}$ at $\beta$. If $f(\beta+n) \neq 0$ for infinitely many $n$, then $K$ admits $f$ at $\beta$. Thus we may assume that $\beta \notin B$, so there is an $m$ so that $f_{C_{1}}(\beta+n)=0$ if $n>m$ and $i>m$, and we may also assume that $f(\beta+n)=0$ for all $n>m$. Since $g_{H}(\alpha)>\dot{g}_{K}(\alpha) \neq 0$ or $F(\alpha) \geqslant f(\alpha) \neq 0$ for some $\alpha \geqslant \beta+\omega$, we have $\Sigma_{n<\omega} F(\beta+m+n)$ infinite. Let $H^{\prime}=\Sigma_{i<m} C_{i}$ and $K^{\prime}=H^{\prime} \cap K$. Then

$$
\sum_{n<\omega} f_{K^{\prime}}(\beta+m+n)=\sum_{n<\omega} f_{K}(\beta+m+n)=\sum_{n<\omega} F(\beta+m+n)
$$


is infinite. So $\Sigma_{n<\omega} f_{C_{i}}(\beta+m+n)$ is infinite for some $i<m$, whence $J_{K} \lambda, \lambda$ $+n) \neq 0$ for some $n$ depending on $\lambda$, for infinitely many $\lambda$ such that $\beta<\lambda<\beta+\omega$.

By Theorem 12, we can embed $K$ nicely in a countable reduced group $G$ such that $G / K$ is torsion and $f_{G, K}=f$. Therefore $G$ is a Warfield group with Ulm function $F$ and Warfield invariants equal to those of $K$, which are equal to those of $H$.

7. The general existence theorem. All the groundwork has now been laid for the fundamental existence theorem for arbitrary Warfield groups.

THEOREM 14. Let $H$ be a direct sum of infinite cyclic reduced valuated groups. Let $F$ be a function from the ordinals to the cardinals that vanishes beyond some ordinal. If $F$ dominates $F+g_{H}$, and $F \geqslant f_{H}$, then there exists a Warfield group $G$ with Ulm function $F$ and Warfield invariants equal to those of $H$. Furthermore $G=A \oplus B$ where $A$ is simply presented, $B$ is a Warfield group, and there is an ordinal $\lambda$ such that $p^{\lambda} B$ is countable and $B / p^{\lambda} B$ is torsion.

Proof. Let $\lambda$ be the least (limit) ordinal such that $\Sigma_{n<\omega} F(\lambda+m+n)$ is countable for some $m<\omega$. Since $F \geqslant f_{H}$ and $F$ dominates $g_{H}$, we can find $K$ subordinate to $H$ such that $K=L \oplus M$ with:

(1) $M=M(\lambda)$ countable,

(2) $f_{M}(\theta)=0$ if $\lambda<\theta<\lambda+m$,

(3) $L(\lambda+\omega)=0$,

(4) $f_{L}(\theta)=0$ if $\theta \geqslant \lambda$.

Let $f(\alpha)=F(\alpha)$ if $\alpha<\lambda$ and $F(\alpha)$ is infinite, and set $f(\alpha)=0$ otherwise. If $\beta<\lambda$, then

$$
\sum_{n<\omega} f(\beta+n)=\sum_{n<\omega} F(\beta+n)
$$

since the right-hand side is uncountable. Let $f^{*}(\alpha)=F(\alpha)$ for $\lambda<\alpha<\lambda+$ $m$, and $f^{*}(\alpha)=0$ otherwise. Then $f+f^{*}$ dominates $f+f^{*}+g_{L}$, so by Theorem 5 , there is a simply presented group $A$ containing $L$ as a nice decomposition basis, with $f_{A, L}=f+f^{*}$. Since $F>f_{H}>f_{K}=f_{L}+f_{M}$ and $f_{L}(\alpha)+f_{M}(\alpha)=0$ for $\lambda \leqslant \alpha<\lambda+m$, we can find a function $f^{\prime}$ such that $F=f_{L}+f_{M}+f^{*}+f^{\prime}$ and $f^{\prime}(\alpha)=0$ for $\lambda \leqslant \alpha<\lambda+m$. Let $F_{B}=f+f_{M}$ $+f^{\prime}$. Note that $F_{B}(\alpha)=f^{\prime}(\alpha)=F(\alpha)$ if $\alpha \geqslant \lambda+m$, and that

$$
\sum_{n<\omega} F_{B}(\beta+n)=\sum_{n<\omega} f(\beta+n)=\sum_{n<\omega} F(\beta+n)
$$

if $\beta<\lambda$. Thus $F_{B}$ dominates $F_{B}+g_{M}$, since $F$ dominates $F+g_{H}$. Define $F_{C}(\alpha)=F_{B}(\lambda+\alpha)$ and let $N$ be $M$ revalued so that $v_{M} x=\lambda+v_{N} x$. Then $F_{C}$ dominates $F_{C}+g_{N}$ and $F_{C}>f_{N}$. Also $F_{C}(\alpha)$ is countable for each $\alpha$, and $F_{C}$ vanishes beyond some countable ordinal (because $F_{B}$ is admissible). 
Therefore, by Theorem 13, there is a countable Warfield group $C$ with Ulm function $F_{C}$ and Warfield invariants equal to those of $N$.

Since $F_{B}$ is admissible, there is a simply presented torsion group $T$ whose Ulm function $f_{T}$ is $F_{B}$ restricted to the ordinals less than $\lambda$. By [1, Theorem 76.1] there is a group $B$ such that $B / p^{\lambda} B \cong T$ and $p^{\lambda} B \cong C$, the latter isomorphism being of groups, not of valuated groups. It is easily seen that $B$ is a Warfield group with Ulm function $F_{B}$ and Warfield invariants equal to those of $M$. Then $G=A \oplus B$ is a Warfield group with Ulm function

$$
\begin{aligned}
f_{A}+f_{B} & =f_{L}+f+f^{*}+f+f_{M}+f^{\prime} \\
& =f+f_{L}+f_{M}+f^{*}+f^{\prime}=f+F=F,
\end{aligned}
$$

and Warfield invariants equal to those of $L \oplus M=K$, and hence to those of $H$.

\section{REFERENCES}

1. L. Fuchs, Infinite abelian groups, Vol. I, Academic Press, New York, 1970. MR 41 \#333.

2. R. Hunter, F. Richman and E. A. Walker, Simply presented valuated abelian p-groups, J. Algebra (to appear).

3. F. Richman, The constructive theory of countable abelian p-groups, Pacific J. Math. 45 (1973), 621-637. MR 49 \#8828.

4. The constructive theory of KT-modules, Pacific J. Math. 61 (1975), 263-274.

5. F. Richman and E. A. Walker, Ext in pre-abelian categories, Pacific J. Math. (to appear).

6.

7. L. A. Rogers, Ulm's theorem for partially ordered structures related to simply presented abelian p-groups, Trans. Amer. Math. Soc. 227 (1977), 333-343.

8. J. Rotman and T. Yen, Modules over a complete discrete valuation ring, Trans. Amer. Math. Soc. 98 (1961), 242-254. MR 23 \#A227.

9. R. O. Stanton, An invariant for modules over a discrete valuation ring, Proc. Amer. Math. Soc. 49 (1975), 51-54. MR 50 \# 13020.

10. E. A. Walker, Ulm's theorem for totally projective groups, Proc. Amer. Math. Soc. 37 (1973), 387-392. MR 47 \#367.

11. R. B. Warfield, Jr., Classification theorems for $p$-groups and modules over a discrete valuation ring, Bull. Amer. Math. Soc. 78 (1972), 88-92. MR 45 \#378.

12. ___ Classification theory of abelian groups. II: Local theory (to appear).

Mathematical Sciences Department, New Mexico State University, Las Cruces, New MEXICO 88003 